\title{
Effects of geometrical characteristics of surface roughness on droplet wetting
}

\author{
Yu-Jane Sheng \\ Department of Chemical Engineering, National Taiwan University, Taipei, Taiwan 106, Republic of China
}

\author{
Shaoyi Jiang \\ Department of Chemical Engineering, University of Washington, Seattle, Washington 98195, USA \\ Heng-Kwong Tsao a) \\ Department of Chemical and Materials Engineering, Institute of Materials Science and Engineering, \\ National Central University, Jhongli, Taiwan 320, Republic of China
}

(Received 10 September 2007; accepted 10 October 2007; published online 19 December 2007)

\begin{abstract}
Surface roughness is known to alter the wettability on a solid substrate. In general, either Wenzel or Cassie-Baxter theory is adopted to describe the apparent contact angle. Following the minimum free energy pathway associated with the imbibition process, we have derived a generalized expression for the apparent contact angle on a textured surface and the liquid-gas contact area within the groove that plays a key role. Depending on the geometrical characteristics of the grooves, the surface wetting falls into three regimes: (i) single stable state which is either Wenzel (completely wetted roughness) or Cassie-Baxter (completely nonwetted roughness) state, (ii) two stable states (Wenzel and Cassie-Baxter) separated by an energy barrier, and (iii) single stable state with partially wetted roughness. The sufficient condition for each regime is derived and several groove geometries are given to show the free energy path. Alteration in the geometric parameters may lead to the wetting crossover. We also show that the Cassie-Baxter can occur at a hydrophilic surface for particular pore shapes. (c) 2007 American Institute of Physics. [DOI: 10.1063/1.2804425]
\end{abstract}

\section{INTRODUCTION}

The wetting of solid surfaces by a liquid (water in particularly) is ubiquitous in everyday lives as well as in industrial processes. Wettability is one of the most important properties associated with a solid surface and the wetting behavior is governed by two factors; the chemical composition and the roughness of the solid surfaces. In terms of the contact angle $\theta$ between the gas-liquid and solid-liquid interfaces, the wettability of an ideal flat solid is depicted by Young's equation, ${ }^{1}$

$$
\cos \theta=\frac{\gamma_{s}-\gamma_{s l}}{\gamma_{l}},
$$

where $\gamma_{s l}, \gamma_{s}$, and $\gamma_{l}$ represent the interfacial tensions of solid-liquid, solid-gas, and liquid-gas interfaces, respectively. In the absence of surface roughness, Young's equation indicates that the nature of wetting is determined by the relative affinity of the solid for the liquid or gas phases, as illustrated by the difference between solid-gas and solid-liquid interfacial tensions in Young's equation. The interfacial tensions $\gamma_{s l}$ and $\gamma_{s}$ are intrinsic properties associated with a surface and they can be controlled by chemical modification, such as fluorination. As $\gamma_{s}-\gamma_{s l}>0$, the contact angle is less than $90^{\circ}$ (hydrophilic surface), whereas $\theta>90^{\circ}$ (hydrophobic surface) for $\gamma_{s}-\gamma_{s l}<0$.

Real solids are actually rough and, thus, their wettability is significantly influenced by the geometrical structure of the

${ }^{a)}$ Electronic mail: hktsao@cc.ncu.edu.tw. surface roughness. The earliest work on the wetting of rough substrates was addressed by Wenzel ${ }^{2}$ and later by Cassie and Baxter. ${ }^{3}$ Wenzel assumed that the liquid filled up the grooves on the rough surface and generalized Young's equation to obtain the apparent contact angle $\theta_{a}$,

$$
\cos \theta_{a}=r \cos \theta,
$$

where $r$ is termed the "roughness factor" and defined as the ratio of the actual area of a rough surface to the geometric, projected area on the horizontal plane. Evidently, the effect of the roughness results in the improvement of the wetting for $\theta<90^{\circ}$ but enhances the hydrophobicity for $\theta>90^{\circ}$. Cassie and Baxter considered the wettability of a composite surface, composed of two types of homogeneous patches that have different solid-fluid interfacial tensions. The apparent contact angle is then given by

$$
\cos \theta_{a}=f_{1} \cos \theta_{1}+f_{2} \cos \theta_{2},
$$

where $f_{i}$ and $\theta_{i}$ represent the surface area fraction and the contact angle of patch $i$, respectively.

For porous or corrugated surfaces, the roughness is mainly filled with air. The openings of the pores can be regarded as nonwetting patches with $\theta_{2}=180^{\circ}$. Since $f_{2}=1$ $-f_{1}$, Eq. (3) becomes

$$
\cos \theta_{a}=f_{1}\left(1+\cos \theta_{1}\right)-1 .
$$

In accord with Eq. (4), if surface hydrophobicity $\left(\theta_{1}\right)$ and surface roughness $\left(f_{1}\right)$ are appropriately combined, a water droplet deposited on such a superhydrophobic surface can remain nearly spherical. A beautiful example is the leaves of 
the lotus plant. Owing to the geometrical structure of solid surfaces alone, the contact angles for water on those surfaces can range from $140^{\circ}$ to as much as $174^{\circ} .{ }^{4,5}$ Recent advent of microfabrication techniques allow us to design microscale structures on a solid surface and thus to control the wettability by roughening it, even without altering any surface chemistry. Inspired by the so-called lotus effect, superhydrophobic surfaces have been created by decorating a homogeneous substrate with an array of pillars. ${ }^{6-8}$

Besides the roughness factor $(r)$ and the wetted area fraction $(f)$, the Wenzel and Cassie-Baxter theories are essentially independent of the geometrical characteristics of the roughness (the shape of the pore). For a given roughness geometry, the Wenzel and Cassie-Baxter theories may predict different apparent contact angles. The wetting state is usually believed to be in either Wenzel or Cassie-Baxter state. However, it is not decisively clear on which theory should be employed and when. A simple criterion is that the wetting state corresponds to the one with a lower free energy. In general, the Wenzel state (wetted groove) prevails for $\theta$ $<\theta_{c}$, while the Cassie-Baxter state (air pocket) dominates for $\theta>\theta_{c}$, where $\theta_{c}$ denotes the critical point and $\theta_{c} \geqslant \pi / 2$. $^{6,8}$ Recently, it is experimentally showed that even if the Wenzel drop possesses the lower energy, a Cassie-Baxter drop can be observed. In fact, there could be two apparent contact angles on the same rough surface, depending on how a drop is formed. ${ }^{6,8}$ For example, when pressing a drop on a hydrophobic surface decorated with spikes, a sharp change in the apparent contact angle from $170^{\circ}$ to $130^{\circ}\left( \pm 5^{\circ}\right)$ is observed. ${ }^{6}$ Another example is that the Cassie-Baxter state is formed if the droplet is deposited gently on a hydrophobic surface with square pillars but the Wenzel state is formed if dropped from a height. ${ }^{8}$ The possibility of multiple free energy minima for droplet states has been proposed to explain the phenomenon of multiple apparent contact angles. ${ }^{8-11}$ Therefore, work must be done to cause the transition from the Cassie-Baxter to Wenzel state because of the energy barrier between them.

The actual details of the crossover from the CassieBaxter to Wenzel state are not well understood and there may exist many pathways. A possible pathway has been considered: the liquid enters the valleys and wets the sides of the pillars by maintaining the location of the liquid-gas interface at the same height from the bottom of the groove (imbibition in parallel). ${ }^{10,11}$ In fact, the extent of penetration into the roughness grooves is initially unknown and has to be determined by the minimization of the free energy. ${ }^{12}$ As a result, the role of the liquid-gas interfaces within the roughness grooves is essential. If the imbibition of liquid into roughness pores in parallel corresponds to the minimum free energy pathway in the free energy landscape, one is able to write down a generalized free energy formulation and finds out the possible stable states for wetting on a textured surface. In this paper, we focus on surfaces with roughness pores and predict the existence of a stable state with partially wetted roughness in addition to the Wenzel (completely wetted roughness) and Cassie-Baxter (completely nonwetted roughness) states. In addition, the influences of the geometrical characteristics associated with roughness pores on the wetting state are examined.

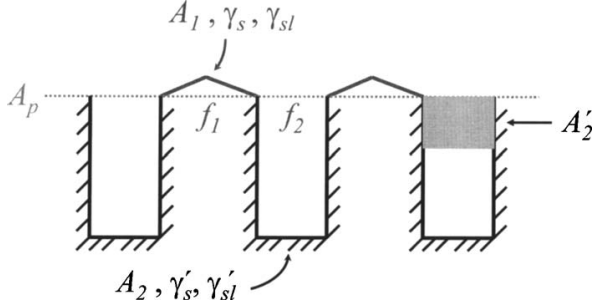

FIG. 1. Schematic representation of a heterogeneous surface containing two types of roughness. The first type is always wetted and possesses actual area $A_{1}$, interfacial tensions $\gamma_{s}$ and $\gamma_{s l}$, and projected area fraction $f_{1}$. The second type can form air pockets (grooves) and has the corresponding properties $\left\{A_{2}, \gamma_{s}^{\prime}, \gamma_{s l}^{\prime}, f_{2}\right\} . A_{2}^{\prime}$ depicts the wetted area within the grooves.

\section{FREE ENERGY FORMULATION}

We start by considering the free energy of a droplet sitting on a rough, chemically heterogeneous substrate. For simplicity, we assume that the radius of the drop is much greater than the separation of asperities. For a sufficient small drop, where the change in hydrostatic pressure with height can be negligible, it can be shown that the solutions of the axisymmetric Laplace equation yield a gas-liquid interface that has the shape of a spherical cap with the radius $R$ of curvature. ${ }^{13}$ Therefore, the free energy can simply be expressed by

$$
F=\pi\left(R \sin \theta_{a}\right)^{2}\left(\gamma_{s l}^{*}-\gamma_{s}^{*}\right)+2 \pi R^{2}\left(1-\cos \theta_{a}\right) \gamma_{l},
$$

where $\gamma_{s l}^{*}$ and $\gamma_{s}^{*}$ denote the effective interfacial tensions for solid-liquid and solid-gas contacts, respectively, due to the heterogeneity of surfaces. Since the volume $V$ of the drop is constant, the drop radius $R$ of curvature is related to apparent contact angle $\cos \theta_{a}$ by

$$
V=\frac{\pi R^{3}}{3}(1-\eta)^{2}(2+\eta)
$$

where $\eta=\cos \theta_{a}$. Equation (6) is justified when the liquid volume within the roughness (pores) is small compared to the total volume. The minimization of the free energy, $\partial F / \partial \eta=0$, yields a relation similar to Young's equation,

$$
\gamma_{l} \cos \theta_{a}=\gamma_{s}^{*}-\gamma_{s l}^{*} \text {. }
$$

In terms of the apparent contact angle, the free energy can be rewritten as

$$
\frac{F(\eta)}{\pi \gamma_{l}}=\left(\frac{3 V}{\pi}\right)^{2 / 3}\left[(1-\eta)^{2}(2+\eta)\right]^{1 / 3} .
$$

Since $F$ decreases monotonically with increasing $\eta$, this result reveals that a droplet with a lower apparent contact angle possesses a lower free energy.

Now the goal is to determine the effective interfacial tensions associated with a composite surface. For a homogeneous surface, one has $\gamma_{i}^{*}=\gamma_{i}$ and Eq. (7) simply reduces to Young's equation. Without loss of generality, we consider a textured surface with two types of roughness. As shown in Fig. 1, the first type of surface (convex surface), possessing actual area $A_{1}$ and interfacial tensions $\gamma_{s}$ and $\gamma_{s l}$, is always wetted by the liquid. The second type of surface (concave surface) is associated with pores or spikes, which may form air pockets. Its actual area and interfacial tensions are de- 
noted by $A_{2}, \gamma_{s}^{\prime}$, and $\gamma_{s l}^{\prime}$, respectively. Note that these two different types of surfaces can have different interfacial tensions. The total projected area is $A_{p}$ and the area fraction of the type $i$ corresponding to $A_{p}$ is $f_{i}$ with $f_{1}+f_{2}=1$. The roughness factor of the type $i$ surface $\left(r_{i}\right)$ is defined as

$$
r_{i}=\frac{A_{i}}{A_{p} f_{i}} \geqslant 1 .
$$

The effective solid-gas interfacial tension (per unit projected area) $\gamma_{s}^{*}$ is simply the sum of the contributions originating from all types of surfaces in the absence of liquid.

$$
\gamma_{s}^{*}=f_{1}\left(r_{1} \gamma_{s}\right)+f_{2}\left(r_{2} \gamma_{s}^{\prime}\right) \text {. }
$$

The effective solid-liquid interfacial tension $\gamma_{s l}^{*}$ is more complicated. It may involve liquid-gas and solid-gas contacts within the air pockets. If the liquid wets the pores partially, then the wetted area of the second type $\left(A_{2}^{\prime}\right)$ can be related to the projected area by $r_{2}^{\prime}=A_{2}^{\prime} / A_{p} f_{2}$ with $0 \leqslant r_{2}^{\prime} \leqslant r_{2}$. In terms of $r_{2}^{\prime}, \gamma_{s l}^{*}$ can be expressed by

$$
\gamma_{s l}^{*}=f_{1}\left(r_{1} \gamma_{s l}\right)+f_{2}\left[r_{2}^{\prime} \gamma_{s l}^{\prime}+\left(r_{2}-r_{2}^{\prime}\right) \gamma_{s}^{\prime}+h\left(r_{2}^{\prime}\right) \gamma_{l}\right] .
$$

Besides the contribution from the first type of surface, the liquid may wet the second type of surface partially with area $A_{2}^{\prime}$ in the air pockets and the rest of the second type of surface with area $\left(A_{2}-A_{2}^{\prime}\right)$ is still in contact with air. When the pores are partially wetted, the liquid is also in contact with air. The liquid-gas contact area within the groove $\left(A_{h}\right)$ is related to the projected area by $h=A_{h} / A_{p} f_{2}$. Note that this scaled liquid-gas contact area $(h)$ may vary with the wetted area in the pores and relates to the minimum free energy pathway along the "wetting coordinate" $r_{2}^{\prime}, 0 \leqslant h\left(r_{2}^{\prime}\right) \leqslant 1$. Owing to the two extreme conditions, nonwetting and complete wetting of the pores, one has $h\left(r_{2}^{\prime}=0\right)=1$ and $h\left(r_{2}^{\prime}\right.$ $\left.=r_{2}\right)=0$.

Knowing $\gamma_{s}^{*}$ and $\gamma_{s l}^{*}$ gives the relation between apparent contact angle and the extent of imbibition, which follows the minimum free energy pathway. Substituting Eqs. (10) and (11) into Eq. (7) yields

$$
\cos \theta_{a}=r_{1} f_{1} \cos \theta+\left[r_{2}^{\prime} \cos \theta^{\prime}-h\left(r_{2}^{\prime}\right)\right]\left(1-f_{1}\right),
$$

where $\cos \theta^{\prime}=\left(\gamma_{s}^{\prime}-\gamma_{s l}^{\prime}\right) / \gamma_{l}$. This generalized expression can be reduced to the Wenzel and the Cassie-Baxter theories. As $\cos \theta^{\prime}=\cos \theta$ and $r_{2}^{\prime}=r_{2}$ (complete wetting of the pores), Eq. (12) becomes

$$
\cos \theta_{a}=\left(\frac{A_{1}+A_{2}}{A_{p}}\right) \cos \theta=r \cos \theta,
$$

which is simply the result of the Wenzel theory. On the other hand, as $r_{2}^{\prime}=0$ (nonwetting of the pores), one has

$$
\cos \theta_{a}=r_{1} f_{1} \cos \theta-\left(1-f_{1}\right) .
$$

If $r_{1}=1$, the above equation reduces to the result of the Cassie-Baxter theory. Equation (14) indicates that the apparent contact angle of a hydrophobic surface is amplified by both the surface roughness of the wetted, convex area and the liquid-air contact at the openings of the air pockets. It contains main characteristics associated with both Wenzel and Cassie-Baxter theories and can be used in both hydro- philic and hydrophobic regions. Equation (12) also reveals that if $r_{2} \cos \theta^{\prime}>-1$ the free energy of the Wenzel state is lower than that of the Cassie-Baxter state. For $r_{2} \cos \theta^{\prime}<$ -1 , one has the opposite result. However, this information alone is not enough to judge what the stable state is.

\section{ENERGY BARRIER AND PARTIALLY WETTED ROUGHNESS}

The importance of the scaled gas-liquid contact area associated with partial wetting of the pores has been disclosed in Eq. (12), which manifests the free energy path associated with the imbibition process. Along the wetting coordinate $r_{2}^{\prime}$, $h\left(r_{2}^{\prime}\right)$ is assumed to follow the minimum free energy pathway from the Cassie-Baxter scenario (nonwetting of the pores and $r_{2}^{\prime}=0$ ) to Wenzel scenario (complete wetting of the pores and $r_{2}^{\prime}=r_{2}$ ) or vice versa. That is, for a simple geometry of roughness such as circular cones, $h\left(r_{2}^{\prime}\right)$ corresponds to the minimum liquid-gas contact area at a given $r_{2}^{\prime}$ (wetting area in the pores). If one knows the wetting/dewetting path and the geometry of the pores, then the relation between $h$ and $r_{2}^{\prime}$ can be determined. Consequently, the free energy path $F\left[\eta\left(r_{2}^{\prime}\right)\right]$ can be obtained for a given set of physical properties $\left\{\theta, \theta^{\prime}, r_{1}, r_{2}, f_{1}\right\}$. After locating the free energy minima or the energy barrier (maximum), the stable droplet shape (or apparent contact angle) can be inferred.

The actual detail of the wetting path is not well understood and it depends on the wetting process. Although different possibilities may be hypothesized, the simplest scenarios for the wetting/dewetting process are wetting/ dewetting all pores in parallel ${ }^{10,11}$ or in series. We assume that imbibition into all pores proceeds in parallel and the effect of meniscus in the grooves is neglected. For a specified geometry of the pores, the variation of the free energy with increasing the wetting area can be evaluated by Eq. (8),

$$
\frac{\partial F}{\partial r_{2}^{\prime}}=\frac{\partial F}{\partial \eta}\left(\frac{\partial \eta}{\partial r_{2}^{\prime}}\right)=-\frac{(1+\eta)}{\left[(1-\eta)(2+\eta)^{2}\right]^{1 / 3}}\left(\frac{\partial \eta}{\partial r_{2}^{\prime}}\right),
$$

where the free energy is scaled by $\pi \gamma_{l}(3 V / \pi)^{2 / 3}$. The possible stable states of wetting can be decided by Eq. (15) for $-1 \leqslant \eta \leqslant 1$. If $\partial F / \partial r_{2}^{\prime} \neq 0$ for $0 \leqslant r_{2}^{\prime} \leqslant r_{2}$, then one has a border minimum corresponding to a single stable state. When $\partial F / \partial r_{2}^{\prime}<0\left(\partial \eta / \partial r_{2}^{\prime}>0\right)$, the Wenzel state is the only stable state because the free energy declines with increasing the wetting area in the roughness. On the contrary, when $\partial F / \partial r_{2}^{\prime}>0\left(\partial \eta / \partial r_{2}^{\prime}<0\right)$, the Cassie-Baxter state is the only stable state since wetting the grooves results in the increment in free energy.

If $\partial F / \partial r_{2}^{\prime}=0$ at $r_{2}^{\prime}=r_{2}^{*}$ with the condition $0<r_{2}^{*}<r_{2}$, then there exists the extremum corresponding to a possible wetting state in the free energy landscape. The stability of the state $F\left(r_{2}^{*}\right)$ has to be determined by the sign of the second derivative. When $\partial^{2} F / \partial r_{2}^{\prime 2}\left(r_{2}^{*}\right)<0, F\left(r_{2}^{*}\right)$ denotes the local maximum and thus both the Wenzel and Cassie-Baxter states are the border minima. That is, there exists two stable states and the energy barrier from the Cassie-Baxter to Wenzel state is $\Delta F_{b}=F\left(r_{2}^{*}\right)-F\left(r_{2}^{\prime}=0\right)$. The energy barrier is able to resist a certain extent of external disturbances and prevents the crossover from one state to another. On the contrary, for 


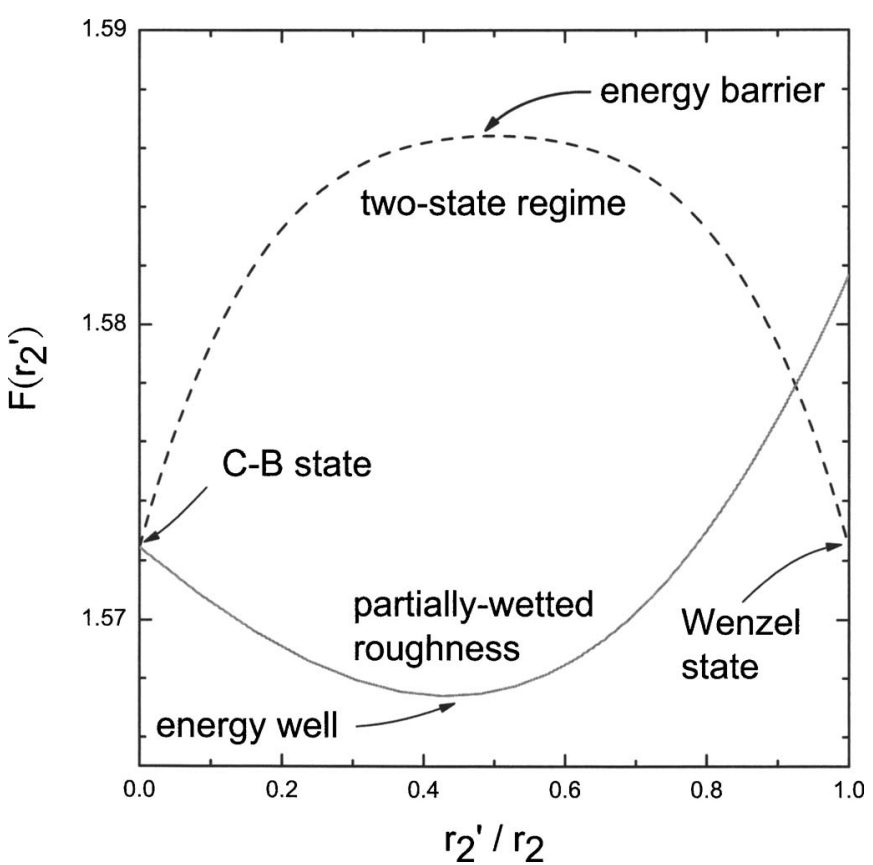

FIG. 2. The minimum free energy pathway along the wetting coordinate $r_{2}^{\prime}$. The free energy is scaled by $\pi \gamma_{l}(3 V / \pi)^{2 / 3}$. The two-state regime with an energy barrier is drawn for hemispherical (convex) pores while the stable state with partially wetted roughness is obtained from concave pores of depth 0.5 , created by the revolution of $y=x^{1 / 3}$ about the $y$ axis. The used parameters are $\cos \theta=\cos \theta^{\prime}=-0.5, r_{1}=1$, and $f_{1}=0.4$.

$\partial^{2} F / \partial r_{2}^{\prime 2}\left(r_{2}^{*}\right)>0, F\left(r_{2}^{*}\right)$ denotes the local minimum and, therefore, the stable wetting state is associated with partially wetted roughness with the penetration extent $0<r_{2}^{*} / r_{2}<1$. This equilibrium state is neither the Wenzel nor CassieBaxter state and only a part of the pore surface is wetted. Two examples for the minimum free energy pathway are illustrated in Fig. 2. The wetting surface with two stable states can be achieved from hemispherical pores while that with partially wetted grooves is obtained from pores created by the revolution of $y=x^{1 / 3}$ about the $y$ axis.

The above analysis indicates that depending on the wetting characteristics of the grooves $h\left(r_{2}^{\prime}\right)$, the wetting state falls into one of the three regimes: (i) single stable state which is either Wenzel or Cassie-Baxter state, (ii) two stable states (Wenzel and Cassie-Baxter) separated by an energy barrier, and (iii) single stable state with partially wetted roughness. Clearly, the "phase diagram" of the wetting states on a textured rough surface has to be determined by the scaled liquid-gas contact area function $h\left(r_{2}^{\prime}\right)$, which is, in turn, dependent on the geometric characteristics associated with the surface roughness. According to Eqs. (12) and (15), the free energy is closely related to $h\left(r_{2}^{\prime}\right)$ through

$$
\frac{\partial \eta}{\partial r_{2}^{\prime}}=\left(1-f_{1}\right)\left(\cos \theta^{\prime}-\frac{\partial h}{\partial r_{2}^{\prime}}\right)
$$

The sufficient condition to have the Wenzel state is $\partial F / \partial r_{2}^{\prime}$ $<0$ at $r_{2}^{\prime}=r_{2}$. According to Eq. (16), one has

$$
\cos \theta^{\prime}>\frac{\partial h}{\partial r_{2}^{\prime}}\left(r_{2}^{\prime}=r_{2}\right)
$$

for the Wenzel regime.

On the other hand, the sufficient condition to have the Cassie-Baxter state is $\partial F / \partial r_{2}^{\prime}>0$ at $r_{2}^{\prime}=0$. Using Eq. (16) leads to

$$
\cos \theta^{\prime}<\frac{\partial h}{\partial r_{2}^{\prime}}\left(r_{2}^{\prime}=0\right)
$$

for the Cassie-Baxter regime.

These two equations indicate that the possible wetting state is the result of the competition between the energy reduction by wetting the surface of the roughness $\left(\cos \theta^{\prime}\right)$ and the energy increment due to the increase of the liquid-gas contact area within the groove $\left(\partial h / \partial r_{2}^{\prime}\right)$. When the former dominates over the latter, one has the Wenzel state. On the contrary, the Cassie-Baxter model is the stable state as the latter is dominant. For a given pore shape, Eqs. (17) and (18) provide the boundaries in the phase diagram. The overlapped domain between the Wenzel and Cassie-Baxter regimes represents the regime with two stable states. Conversely, the domain, which is neither the Wenzel or Cassie-Baxter regimes, denotes the stable state with partially wetted roughness.

\section{EXAMPLES OF PHASE DIAGRAM}

The phase diagram is determined by the liquid-gas contact area within the grooves $h\left(r_{2}^{\prime}\right)$, which is a function of the geometrical characteristic of the roughness. In general, the shape of the pore can simply be classified into linear (e.g., circular cone), convex (e.g., hemisphere), or concave (e.g., revolution of asteroid) functions. In order to demonstrate the three regimes, we consider an example for each case.

\section{A. Single stable state: Wenzel or Cassie-Baxter}

We consider a smooth surface with linear pores, which are modeled as circular cones. The radius of the opening is $a$ and the depth is $h$. We assume that $r_{1}=1$ and the area fraction occupied by pores is $1-f_{1}$. The total second type surface area is represented by the roughness factor $r_{2}=\pi a l / \pi a^{2}>1$, where $l=\left(a^{2}+h^{2}\right)^{1 / 2}$. When the liquid-gas contact area in the pore is depicted by a circle with radius $b$, the wetted area within the grooves is $r_{2}^{\prime}=\pi a l(1+b / a)(1-b / a) / \pi a^{2}=[1$ $\left.-(b / a)^{2}\right] r_{2}$. As a result, the liquid-gas contact area is

$$
h\left(r_{2}^{\prime}\right)=\left(\frac{b}{a}\right)^{2}=1-\left(\frac{r_{2}^{\prime}}{r_{2}}\right) \text {. }
$$

According to Eq. (12), the apparent contact angle is

$$
\cos \theta_{a}=f_{1} \cos \theta+\left[r_{2}^{\prime}\left(\cos \theta^{\prime}+\frac{1}{r_{2}}\right)-1\right]\left(1-f_{1}\right),
$$

which varies linearly with the wetting area $r_{2}^{\prime}$ with the slope $\left(1-f_{1}\right)\left(\cos \theta^{\prime}+1 / r_{2}\right)$. Since $\partial \eta / \partial r_{2}^{\prime} \neq 0$, there exists no extreme value for $0 \leqslant r_{2}^{\prime} \leqslant r_{2}$. As a result, only one stable state (either at $r_{2}^{\prime}=0$ or $r_{2}^{\prime}=r_{2}$ ) is possible and the stable state (minimum free energy) is determined by the value of 


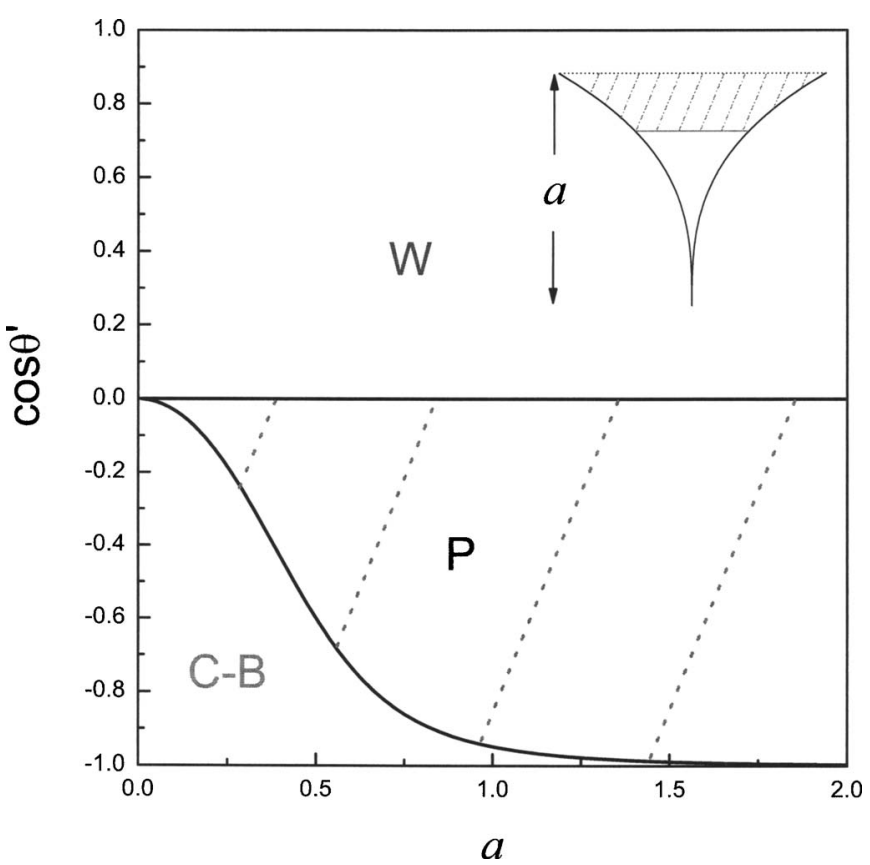

FIG. 3. The phase diagram for concave pores created by the revolution of $y=x^{1 / 3}$ about the $y$ axis. Depending on the intrinsic contact angle of the roughness $\left(\cos \theta^{\prime}\right)$ and the depth of the pore $(a)$, the wetting state may be in the regime of Wenzel (W), Cassie-Baxter (CB), or partially wetted roughness $(P)$. The two-state regime does not exist for such geometry. The phase boundaries are determined by Eqs. (17) and (18).

$\left(r_{2} \cos \theta^{\prime}+1\right)$. As $r_{2} \cos \theta^{\prime}<-1, r_{2}^{\prime}=0$ yields the lowest free energy (Cassie-Baxter state). On the contrary, for $r_{2} \cos \theta^{\prime}$ $>-1, r_{2}^{\prime}=r_{2}$ corresponds to the largest apparent contact angle (Wenzel state). That is, even for hydrophobic pores $\left(\cos \theta^{\prime}\right.$ $<0$ ), the pores can be completely wetted as long as the pore is shallow enough (small $l$ ). If $\cos \theta^{\prime}=-1 / r_{2}$, the apparent contact angle maintains the same regardless of the wetting area. This consequence indicates that for a given $\cos \theta^{\prime}<0$, the Cassie-Baxter state can be achieved by increasing $r_{2}$. For example, increasing the depth of the pore $(h)$ large enough leads to the formation of air pockets. Nevertheless, the highest apparent contact angle that can be achieved depends on the area fraction of solid surface $f_{1}$, i.e., $\cos \theta_{a}=f_{1} \cos \theta$ $-\left(1-f_{1}\right)$. Note that for such a geometry with a given $r_{2}$ or $\{a, h\}$, it is impossible to observe the crossover between the Cassie-Baxter and Wenzel states by external disturbances.

\section{B. Single stable state: partially wetted roughness}

When the liquid-gas contact area in the pore declines fast enough with increasing the wetting area, the minimum free energy may take place at neither complete nonwetting nor complete wetting of the pores. In the second case, we consider a smooth surface with concave pores. They are created by the revolution of $y=c^{2 / 3} x^{1 / 3}$ about the $y$ axis, as depicted in Fig. 3. The depth of the pore is $a c$. The analysis is simplified if all lengths are scaled by $c$, i.e. $y=x^{1 / 3}$ with depth $a$. In terms of roughness factor, the area of the second type of surface is $r_{2}=\left[\left(1+9 a^{4}\right)^{3 / 2}-1\right] / 27 a^{6}$. Since imbibition into all pores in parallel is a reasonable assumption, the liquid-gas contact area is

$$
\begin{aligned}
h\left(r_{2}^{\prime}\right)= & \left\{\left[1+\left(\left(1+9 a^{4}\right)^{3 / 2}-1\right)\right.\right. \\
& \left.\left.\times\left(1-\frac{r_{2}^{\prime}}{r_{2}}\right)\right]^{2 / 3}-1\right\}^{3 / 2} / 27 a^{6} .
\end{aligned}
$$

According to Eq. (16), the solution $\left(0<r_{2}^{*}<r_{2}\right)$ satisfying $\cos \theta^{\prime}=\partial h / \partial r_{2}^{\prime}\left(\partial F / \partial r_{2}^{\prime}=0\right)$ gives an extremum of the free energy at

$$
r_{2}^{*}=r_{2}-\left[\left(\sin \theta^{\prime}\right)^{-3}-1\right] / 27 a^{6} .
$$

The stability of this state can be examined by $\partial^{2} \eta / \partial r_{2}^{\prime 2}<0$ $\left(\partial^{2} F / \partial r_{2}^{\prime 2}>0\right)$ at $r_{2}^{*}$. Since the second derivative of $\eta$ at $r_{2}^{*}$ is given by

$$
\frac{\partial^{2} \eta}{\partial r_{2}^{\prime 2}}=\left(1-f_{1}\right)\left(-\frac{\partial^{2} h}{\partial r_{2}^{\prime 2}}\right)=-\left(1-f_{1}\right) 9 a^{6} \frac{\sin ^{3} \theta^{\prime}}{\left|\cos \theta^{\prime}\right|}<0,
$$

this extremum corresponds to a minimum of the free energy and a maximum of the apparent contact angle. This result indicates the stable, wetting state with partially wetted roughness. According to Eqs. (17) and (18), the Wenzel and Cassie-Baxter regimes are $\cos \theta^{\prime}>0$ and $\cos \theta^{\prime}<-3 a^{2} /(1$ $\left.+9 a^{4}\right)^{1 / 2}$, respectively. The wetting state depends mainly on the contact angle of the pore $\left(\cos \theta^{\prime}\right)$ and the geometrical parameter $(a)$. The phase diagram is shown in Fig. 3. For hydrophilic surfaces $\left(\cos \theta^{\prime}>0\right)$, the Wenzel state is preferred. For hydrophobic surfaces $\left(\cos \theta^{\prime}<0\right)$, however, the Cassie-Baxter state exists only for shallow pores. The roughness becomes partially wetted for deep apertures.

\section{Two stable states: Wenzel and Cassie-Baxter}

In the third case, we consider square pillars of size $2 a$, height $H$, and spacing $2 b$ arranged in a regular array. The pillar is based on a square pyramid with slant height $l(>b)$ as illustrated in Fig. 4. This geometry can be regarded as convex pores. Assume that $r_{1}=1$ and the area fraction associated with the top surface of the pillar is $f_{1}$. The area of the second type of surface is $r_{2}=2 a H /\left[(a+b)^{2}-a^{2}\right]+l / b$. Assume imbibition into grooves in parallel and then the liquid-gas contact area is

$$
h\left(r_{2}^{\prime}\right)= \begin{cases}1, & \text { for } x \leqslant H \\ {\left[1+\frac{2 a+b}{l}\left(r_{2}^{\prime}-r_{2}+\frac{l}{b}\right)\right]\left[1-\frac{b}{l}\left(r_{2}^{\prime}-r_{2}+\frac{l}{b}\right)\right],} & \text { for } x>H,\end{cases}
$$




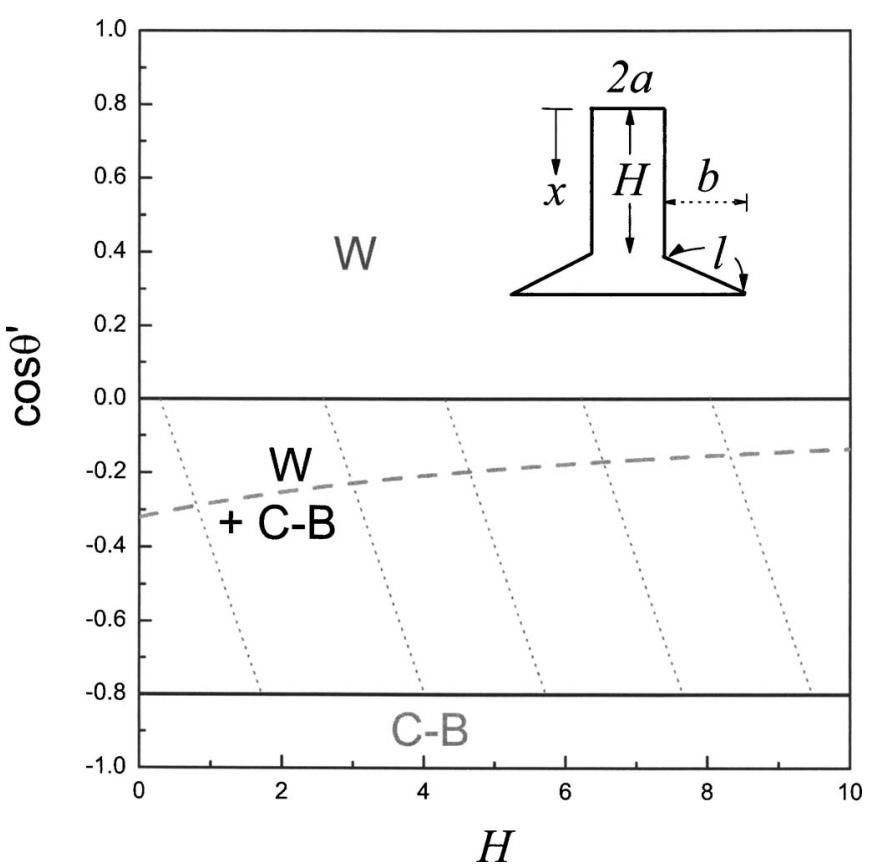

FIG. 4. The phase diagram for convex grooves created by square pillars based on square pyramids. There exists a two-state regime where both the Wenzel (W) and Cassie-Baxter (CB) states are stable. The dashed line depicts the boundary for the state with lower free energy. External work is required for the wetting transition from the Cassie-Baxter to Wenzel state. The boundary between the Cassie-Baxter and two-state regimes can be moved toward smaller contact angle by decreasing $(a+b) / l$. The used parameters are $a=0.2, b=0.8$, and $l=2.5$.

where $x$ is the depth.

The extremum of the free energy is determined by $\cos \theta^{\prime}=\partial h / \partial r_{2}^{\prime}$ and takes place at

$$
r_{2}^{*}=\left[2 a H+l\left(a-l \cos \theta^{\prime} / 2\right)\right] /(2 a+b) b .
$$

The stability of this state is examined by $\partial^{2} F / \partial r_{2}^{\prime 2}$ at $r_{2}^{*}$. Since the second derivative of $\eta$ at $r_{2}^{*}$ is

$$
\frac{\partial^{2} \eta}{\partial r_{2}^{\prime 2}}=\left(1-f_{1}\right)\left(-\frac{\partial^{2} h}{\partial r_{2}^{\prime 2}}\right)=2\left(1-f_{1}\right) \frac{(2 a+b) b}{l^{2}}>0,
$$

this extreme corresponds to a maximum of the free energy and a minimum of the apparent contact angle. This consequence indicates that this state is unstable and represents the energy barrier between the two border minima $\left(r_{2}^{\prime}=0\right.$ and $r_{2}^{\prime}=r_{2}$ ). From Eqs. (17) and (18), the Wenzel and CassieBaxter regimes are $\cos \theta^{\prime}>-2(a+b) / l$ and $\cos \theta^{\prime}<0$, respectively. The overlapped domain corresponds to the wetting regime with the two stable states. The result discloses that the pillar height $H$ has no direct effect on the phase diagram. However, in the two-state regime, $r_{2} \cos \theta^{\prime}$ declines with increasing $H$ and, therefore, the Cassie-Baxter state dominates the lower energy state. The external disturbance may lead to the crossover from the Cassie-Baxter to Wenzel state. If one wants to avoid the wetting crossover due to external work, the single Cassie-Baxter regime can be obtained by decreasing $(a+b) / l$.

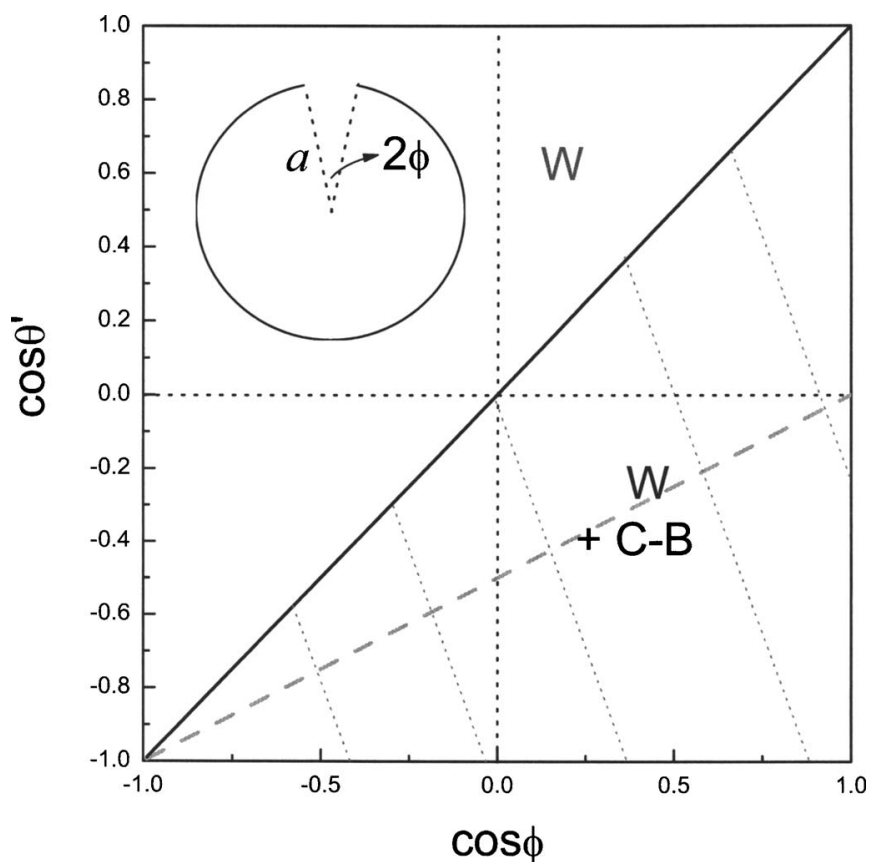

FIG. 5. The phase diagram for spherical cap pores in terms of the opening angle $\phi$ and contact angle $\theta^{\prime}$. For such roughness the two-state regime can happen at all contact angles by tuning the opening angle. The Cassie-Baxter state (CB) may occur at hydrophilic surfaces for $\cos \phi>0$ while the Wenzel state (W) can take place at very hydrophobic surfaces for $\cos \phi<0$.

\section{COULD THE CASSIE-BAXTER STATE OCCUR AT HYDROPHILIC ROUGHNESS?}

Typically, the liquid-gas contact area within the roughness grooves declines with increasing the wetting area and, thus, $\partial h / \partial r_{2}^{\prime}\left(r_{2}^{\prime}=0\right) \leqslant 0$ with $1 \geqslant h\left(r_{2}^{\prime}\right) \geqslant 0$. For such roughness, Eq. (18) reveals that the Cassie-Baxter state always takes place at hydrophobic surfaces $\left(\cos \theta^{\prime} \leqslant 0\right)$. In fact, it is generally believed that the Cassie-Baxter state can occur only at hydrophobic roughness. However, on the basis of Eq. (18), one can design a particular pore shape so that the Cassie-Baxter state occurs even for hydrophilic pores. That is, one must satisfy the criterion $\partial h / \partial r_{2}^{\prime}\left(r_{2}^{\prime}=0\right)>0$ to obtain the completely nonwetted roughness condition for hydrophilic grooves $\left(\cos \theta^{\prime}>0\right)$. For $\cos \theta^{\prime}>0$, the condition $\partial h / \partial r_{2}^{\prime}>0$ reveals that the liquid-gas contact area within the pores rises with increasing the wetting area $r_{2}^{\prime}$. In other words, the Cassie-Baxter state can occur at hydrophilic roughness only when $h\left(r_{2}^{\prime}\right)>1$ at $r_{2}^{\prime} \rightarrow 0^{+}$. A simple example is spherical cap pores, as illustrated in Fig. 5. The radius of the opening is $a \sin \phi$ and the area of the second type surface is $r_{2}=2(1+\cos \phi) / \sin ^{2} \phi$. The liquid-gas contact area is then given by

$$
h\left(r_{2}^{\prime}\right)=\frac{1-\left[\cos \phi-(1+\cos \phi) \frac{r_{2}^{\prime}}{r_{2}}\right]^{2}}{\sin ^{2} \phi} .
$$

According to Eq. (18), the criterion for the Cassie-Baxter regime yields 


$$
\cos \theta^{\prime}<\frac{2}{r_{2}} \frac{\cos \phi(1+\cos \phi)}{\sin ^{2} \phi}=\cos \phi .
$$

This result indicates that the Cassie-Baxter state can occur for hydrophilic grooves in the two-state regime for $\phi<90^{\circ}$, as illustrated in Fig. 5. Conversely, the Wenzel state may persist for very hydrophobic grooves as $\phi>90^{\circ}$. Our result reveals that it is possible to increase the apparent contact angle of a surface with hydrophilic pores by the help of specially designed roughness, which can sustain the CassieBaxter state. Our analysis agrees with the fact that water cannot enter many soils (a particle bed) unless the contact angle is considerably lower than $90^{\circ}$, down to approximately $50^{\circ}$, if the average contact angle between the soil and water is $90^{\circ}$ or lower. ${ }^{14}$ Recently, similar phenomena have been observed for an aqueous surfactant drop on a porous surface. ${ }^{15}$

\section{CONCLUSION}

The free energy of a droplet sitting on a textured surface can be expressed in terms of the effective interfacial tensions. The minimization of the free energy yields a relation similar to Young's equation, which relates the apparent contact angle to the effective interfacial tensions. We have considered a textured surface with two types of roughness. The first type of surface is always wetted by the liquid while the second type of surface representing pores or spikes is able to form air pockets. The effective interfacial tensions can then be related to the liquid-gas contact area within the grooves, which plays an essential role in determining the wetting state of a droplet.

Our analyses have shown clearly that the wetting state of a droplet on a textured rough surface depends on the geometrical shape of the grooves (such as linear, convex, and concave pores) in addition to the intrinsic contact angle. The wetting state may fall into single stable state regime (com- pletely wetted roughness, completely nonwetted roughness, or partially wetted roughness) or two stable states regime (Wenzel or Cassie-Baxter) with an energy barrier separating them. On the basis of imbibition into pores in parallel and following the minimum free energy pathway, the sufficient condition for each regime is derived and the phase diagram can be obtained for a specified roughness. In general, the linear pores exhibit single stable state. The convex pores give two stable states while the concave pores yield single stable state with partially wetted roughness. Our study also suggests that the Cassie-Baxter state may form at a hydrophilic surface with suitably designed grooves.

\section{ACKNOWLEDGMENTS}

This research work is financially supported by BASF Electronic Materials Taiwan Ltd. and National Science Council of Taiwan.

${ }^{1}$ P. G. de Gennes, F. Brochard-Wyart, and D. Quéré, Capillarity and Wetting Phenomena, Drops, Bubbles, Pearls, Waves (Springer, New York, 2004).

${ }^{2}$ R. N. Wenzel, Ind. Eng. Chem. 28, 988 (1936).

${ }^{3}$ A. B. D. Cassie and S. Baxter, Trans. Faraday Soc. 40, 546 (1944),

${ }^{4}$ T. Onda, S. Shibuichi, N. Satoh, and K. Tsujii, Langmuir 12, 2125 (1996).

${ }^{5}$ S. Shibuichi, T. Onda, N. Satoh, and K. Tsujii, J. Phys. Chem. 100, 19512 (1996)

${ }^{6}$ J. Bico, C. Marzolin, and D. Quéré, Europhys. Lett. 47, 220 (1999).

${ }^{7}$ J. Bico, C. Tordeux, and D. Quéré, Europhys. Lett. 55, 214 (2001).

${ }^{8}$ B. He, N. A. Patankar, and J. Lee, Langmuir 19, 4999 (2003).

${ }^{9}$ N. A. Patankar, Langmuir 19, 1249 (2003).

${ }^{10}$ N. A. Patankar, Langmuir 20, 7097 (2004).

${ }^{11}$ C. Ishino, K. Okumura, and D. Quéré, Europhys. Lett. 68, 419 (2004).

${ }^{12}$ A. Marmur, Langmuir 19, 8343 (2003).

${ }^{13}$ R. J. Hunter, Foundations of Colloid Science I (Oxford University Press, New York, 1991).

${ }^{14}$ N. Shirtcliffe, G. McHale, M. I. Newton, F. B. Pyatt, and S. H. Doerr, Appl. Phys. Lett. 89, 094101 (2006).

${ }^{15}$ F.-M. Chang, Y.-J. Sheng, H. Chen, and H.-K. Tsao, Appl. Phys. Lett. 91, 094108 (2007). 